\title{
EL SISTEMA DE EVALUACIÓN DE PROFESORES EN LA PONTIFICIA UNIVERSIDAD JAVERIANA BOGOTÁ, COLOMBIA
}

\author{
Pedro P. Polo Verano*
}

Entre las diversas acciones emprendidas por la Universidad en los últimos diez años, encaminadas al logro de altos niveles de calidad, debe destacarse lo relativo al sistema de evaluación de profesores. Este se enmarca en el sistema general de evaluación en la Universidad, que persigue fundamentalmente el mejoramiento y no el control. El sistema de evaluación de profesores incluye la evaluación de desempeños académicos y administrativos, la evaluación de sus obras de producción intelectual y la evaluación de ciertos logros, tales como la obtención de segundos títulos universitarios, la realización de cursos formales no conducentes a título, la obtención de distinciones académicas y la competencia en idiomas no nativos. A través del conocimiento que se genera en los procesos de evaluación y de los incentivos establecidos en el Reglamento del Profesorado, los profesores y la institución han tomado acciones de mejoramiento que han llevado a un nuevo perfil del profesorado en la Universidad. La dedicación de los profesores ha aumentado notablemente lo cual ha permitido la conformación de una comunidad profesoral científica y estable; la composición del cuerpo profesoral ha mejorado sustancialmente en lo relativo a titulos de posgrado; la producción intelectual se ha incrementado enormemente; y se han multiplicado los logros de los profesores.
During the last ten years, the University has carried out several actions aimed at reaching high quality levels. One of the most important actions taken deals with the system for professor evaluation, which is also part of the general evaluation system implemented in the university not to seek control but to make all the necessary improvements. The professor evaluation system assesses academic and administrative performance, intellectual production and other achievements such as additional university degrees, participation in training courses, academic awards, and proficiency in other languages.

By means of the information obtained from evaluation processes and the incentives established in the Professor Regulations, both the scholars and the institution have implemented improvement actions that have created a new professor profile within the University: their dedication has increased considerably, thus allowing the establishment of a stable scientific teaching community; the teaching staff now has better and more graduate degrees; there is more intellectual production; and their awards have also increased substantially.

* Asistente del Vicerrector Académico. Pontificia Universidad Javeriana, Bogotá, Colombia. 


\section{Antecedentes}

La Ponvificia Universidad Javeriana es una institución colombiana de educación superior de naturaleza privada, pero sin ánimo de lucro que, por mandato constitucional y por ley de la República, desempeña un papel de servicio público al país.

En la Universidad Javeriana, la calidad es el marco dentro del cual se orientan sus acciones para beneficio de la sociedad en general. La institución fue fundada en 1622 , pero nunca ha querido descansar en su largo y bien ganado prestigio. La preocupación por la calidad la llevó a plantearse, a comienzos de la década de los años noventas, la realización de su planeación estratégica. Esta implicó la revisión a fondo de su ser y quehacer, de su Misión y Proyecto Institucional, de su organización, de sus procesos académicos y administrativos, así como de sus recursos humanos, físicos y financieros.

Por otra parte, en el marco de la autonomía universitaria de la cual por primera vez en la historia de Colombia se había ocupado un año antes la Constitución Política, se decidió en el país en 1992, con la expedición de la nueva ley de educación superior (Ley 30 de 1992), abandonar los esquemas excesivamente intervencionistas que, en desarrollo de la anterior legislación, habían venido rigiendo en Colombia desde 1980 hasta ese momento para garantizar la calidad de las instituciones y programas en la educación superior. Entre muchas otras cosas, la nueva Ley introdujo, a cambio de los existentes esquemas de excesiva intervención y control, una política de fomento a la alta calidad a través de un Sistema Nacional de Acreditación, de carácter voluntario y temporal.

Lo anterior llevó a la Universidad Javeriana y a otras nueve universidades colombianas a conformar un grupo de colaboración -lo que se ha denominado el "Grupo de las 10" - en preparación para enfrentar los procesos de la acreditación.

Dado que es demostrable que en una institución de educación superior al menos las dos terceras partes de la varianza de la calidad se deben al profesorado, la Universidad tomó la determinación de poner especial empeño en lograr un cuerpo profesoral científico, estable y de mucha calidad.

El diagnóstico sobre el profesorado no era muy alentador a comienzos de los años noventas. La Javeriana, igual que las demás universidades privadas líderes en el país, tenía una proporción muy baja de profesores de planta (tiempo completo y medio tiempo) frente al número de profesores contratados por horas.

1 El "gnupo de las 10" está integrado por las diez universidades colombianas que se reconocen mutuamente como las instituciones de educación superior de más alto desarrollo. Cuatro de las diez son universidades oficiales y seis son privadas. Todas las universidades en Colombia, públicas y privadas, son de servicio público. Las universidades privadas son entidades sin ánimo de lucro. 
El porcentaje de profesores con doctorado era exiguo y el de profesores con maestría muy bajo. Las publicaciones en Ciencia y Tecnología eran relativamente pocas. No existían incentivos en la Universidad para que el profesorado tuviera una mayor dedicación (los sueldos eran muy bajos), ni para la obtención de tulos de posgrado por parte de los profesores, ni para su producción intelectual.

Ante este panorama, la Universidad dedicó especial interés, dentro de su planeación estratégica, a la modificación significativa del perfil de su cuerpo profesoral. Esto implicó varias acciones:

a) La elaboración de un Reglamento del Profesorado. Este Reglamento define con claridad todas las relaciones entre los profesores y la institución.

b) La conformación de un escalafón profesoral dentro de dicho Reglamento. El escalafón contempla categorías que dependen de los títulos universitarios de los profesores, de su producción intelectual y de su evaluación, y no solamente de la antiguiedad. Contiene reglas claras para la promoción de los profesores de una categoría a otra. Los salarios de los profesores, a su vez, están determinados por la categoría del profesor en el escalafón.

c) La creación de incentivos para la producción intelectual y para la formación de los profesores. La formación de profesores se refiere a la obtención de títulos de posgrado, especialmente de maestría y doctorado, y también a la capacitación de profesores en diferentes temáticas, entre ellas, pedagogía, idiomas, gestión y problemas del contexto.

d) La mejora sustancial de los salarios de los profesores. No solamente se mejoraron los salarios sino que se estableció la oportunidad de escalar a nuevos niveles de remuneración de acuerdo con los ascensos en el escalafón, así como la oportunidad de obtener, por vías de producción intelectual, bonificaciones adicionales a su salario.

e) El aseguramiento de mayores recursos financieros. Se requerían mayores recursos para hacer frente a los aumentos salariales y a las mejoras de las condiciones de trabajo en la institución. Para lo anterior fue necesario lograr un mejor manejo financiero, un incremento de la investigación, de la consultoría y de la venta de otros servicios, así como la creación de empresas productivas, tales como cafeterías, tienda javeriana, centro editorial, centro gráfico, centro de elaboración de medios audiovisuales y agencia de turismo que, manejadas empresarialmente, prestan un buen servicio a la comunidad educativa y generan rendimientos financieros para apoyo de la actividad académica.

f) La creación y puesta en marcha de un sistema de evaluación de profesores. Este sistema está inscrito en el marco del sistema general de evaluación de la Universidad. 
De esas acciones, la definición y la puesta en ejecución del sistema de evaluación de profesores son los objetivos de este arúculo. Se detallan a continuación.

\section{Objetivos}

El sistema de evaluación de los profesores se inscribe en el sistema general de evaluación de la Universidad y forma parte del Reglamento del Profesorado.

Las evaluaciones en la institución buscan siempre y fundamentalmente el mejoramiento de la calidad. El propósito principal que justifica su existencia no es el de controlar o fiscalizar, sino el de aportar a la Universidad como un todo y a cada una de las dependencias implicadas en cada caso, así como a los profesores, la información necesaria sobre sus fortalezas y debilidades con el fin de que se puedan implementar acciones de mejoramiento. Ha sido tarea importante en la Universidad aclarar este concepto. De esta manera se vencen las usuales resistencias a la evaluación y se crea un clima de aceptación y confianza.

En la medida en que las evaluaciones conducen al mejor aprovechamiento de las fortalezas institucionales y de las personas que componen la comunidad universitaria, así como a la corrección de debilidades, y por ende conducen también a las acreditaciones de programas y a las posibilidades de ascenso en el escalafón profesoral y de promoción en el caso de personal administrativo, se logra una disposición muy favorable de las personas a su evaluación personal y a la evaluación de las dependencias y programas en que se desempeñan.

El objetivo fundamental del Reglamento del Profesorado es el de estimular y orientar el desarrollo humano y profesional de los profesores para consolidar una comunidad científica y profesoral estable con el propósito final de lograr la excelencia académica en la Universidad ${ }^{2}$. En consecuencia, el objetivo fundamental de la evaluación de los profesores es el de contribuir al mejoramiento de su desarrollo humano y el de sus funciones en la Universidad. También es objetivo fundamental el mejoramiento de las condiciones institucionales para el mejor desarrollo de las actividades académicas de los profesores. Un aporte adicional, importante para reforzar los objetivos fundamentales, es el de contribuir a las posibilidades de ascenso de los profesores en el escalafón, por medio de la asignación de puntos cuando se logran desempeños "excelentes" y "buenos", así como por la asignación de puntos por producción intelectual y por otros logros de ellos.

2 Esto está tomado del Artículo $1^{\circ}$ del Reglamento del Profesorado, versión de noviembre de 2000. El Reglamento y lo relativo a las evaluaciones de profesores y directivos están publicados en la página web de la Universidad (www.javeriana.edu.co) en Información Institucional, Vicerrectoría Académica, Profesores. 


\section{Tipos de evaluaciones}

En el sistema de evaluación de profesores se distinguen los siguientes cuatro tipos de evaluación:

a) La evaluación del desempeño académico de cada uno de los profesores.

b) La evaluación del desempeño administrativo de los profesores que ocupan cargos directivos.

c) La evaluación de las obras de producción intelectual de cada profesor.

d) La evaluación de otros logros de cada profesor, tales como la obtención de segundos títulos universitarios, la realización de experiencias posdoctorales, la participación certificada en cursos formales no conducentes a títulos, la competencia en idiomas no nativos, la obtención de distinciones académicas y la traducción de las obras de producción intelectual del profesor a otros idiomas, realizada por terceros.

A través de estos diferentes tipos de evaluación se pretende incentivar al profesor para que cualifique a lo largo de toda su carrera académica, su desempeño y sus logros. En la figura 1 se resume el sistema de evaluación que se detalla en las siguientes secciones.

\section{Evaluación de desempeño académico y resultados}

\section{Agentes y periodicidad}

Compete a los decanos de cada Facultad y al director del departamento correspondiente, la evaluación de desempeño académico de los profesores.

Cada profesor de planta debe ser evaluado al menos una vez cada dos años. La Facultad puede establecer una periodicidad más corta. Por iniciativa de los decanos, o del director del departamento al cual está adscrito el profesor, o del mismo profesor, puede realizarse una evaluación de desempeño académico antes de que se cumpla el período establecido. Usualmente esto ocurre cuando el profesor está cercano a satisfacer todos los requisitos para su ascenso en el escalafón o cuando existe algún indicio de razones que ameriten algún plan de mejoramiento específico.

Las evaluaciones periódicas de desempeño académico se iniciaron hace seis años, después de que se realizaron varias pruebas piloto exitosas. Dos años después de su implementación inicial, sin embargo, se realizó una metaevaluación que condujo a nuevos ajustes en los instrumentos y en su aplicación. 


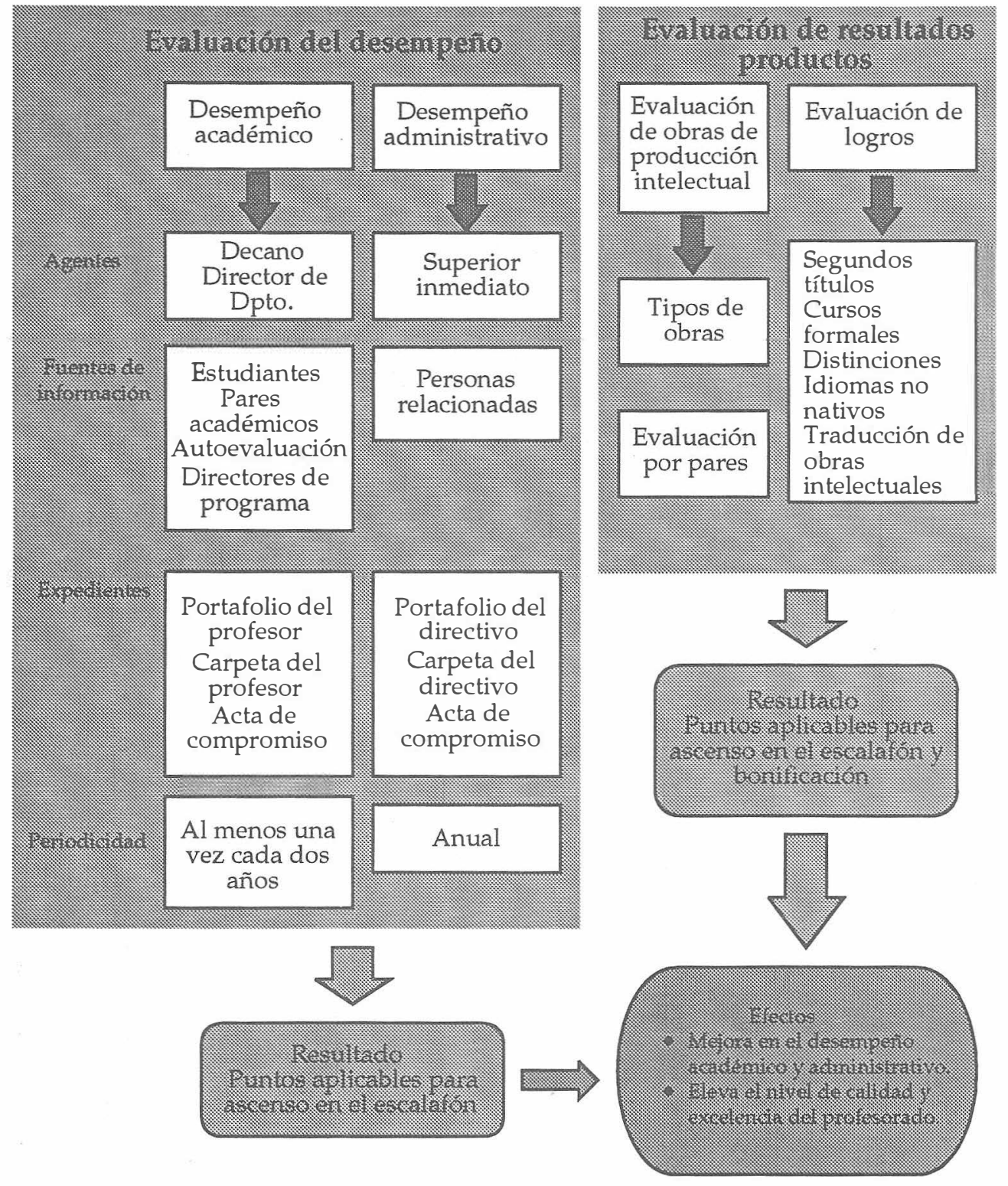

Figura 1. Sistema de Evaluación de Profesores. Pontificia Universidad Javeriana. Bogotá.

\section{Información e instrumentos}

Para la realización de la evaluación periódica de desempeño académico, los decanos y el director del departamento cuentan con información que proviene de diferentes fuentes. 
a) La información que suministran sobre el profesor los estudiantes en cada asignatura a su cargo. Para esto se ha diseñado un formulario muy sencillo de contestar ${ }^{3}$, que contempla solamente diez criterios. Cada criterio, a su vez, está definido por medio de unos cuatro o cinco indicadores que le aclaran al estudiante el alcance del criterio ${ }^{4}$. El estudiante señala para cada criterio si en su opinión el profesor ha sido "excelente", "bueno", "regular" o "malo". También existe la opción de que el estudiante conteste que no sabe. Adicionalmente, en el formulario se da la oportunidad para que, en forma opcional, el estudiante señale alguno o algunos de los indicadores asociados al criterio en el cual (o en los cuales) el profesor especialmente se destaca o especialmente debe hacer un esfuerzo por mejorar. Así, es posible para el estudiante indicar que el profesor es "excelente" o "bueno" en algún criterio (por ejemplo, en el cumplimiento oportuno de sus responsabilidades) y, a pesar de ello, que debe mejorar en algún indicador específico asociado al mismo criterio (por ejemplo, en la entrega oportuna de calificaciones). Los resultados de este instrumento se han constituido en un arma poderosa para dar información a los decanos y a los directores de departamento sobre el profesor evaluado. En los reportes que consolidan la información sobre un profesor, por asignatura dictada, así como por el conjunto de grupos de estudiantes pertenecientes a cada asignatura y por el conjunto de estudiantes del profesor de todas las asignaturas a su cargo, se indica el porcentaje de alumnos que, en cada criterio, han señalado que el profesor es "excelente", "bueno", "regular" o "malo". Adicionalmente, estos reportes señalan aquellos indicadores en que hay una confluencia de opiniones positivas o negativas sobre el profesor. En dichos reportes solamente aparecen aquellos indicadores en que hay coincidencia de opinión positiva o negativa de al menos el $27 \%$ de los estudiantes -esta cifra, definida intuitivamente, ha probado ser muy útil para cernir opiniones individuales y destacar solamente lo relevante-.

b) La información que sobre el profesor aportan los pares académicos del profesor. Para el efecto se ha diseñado otro formulario, semejante al que se

3 El tiempo promedio que le toma a un estudiante contestar el formulario es de 5 minutos. El tiempo máximo es de 8 minutos.

4 Un ejemplo de criterio e indicadores asociados es: "El profesor muestra destreza para motivar el aprendizaje del estudiante, mediante los siguientes indicadores:

A- El estímulo del interés o curiosidad por los temas tratados

B- El fomento en el estudiante de la confianza en sí mismo para asimilar y elaborar el conocimiento

C- La habilidad para generar la participación del estudiante

D. La atención a los estudiantes fuera de clase". 
llena por parte de los estudiantes. Algunos de los criterios sobre los que se pregunta la opinión de los pares son iguales a algunos de los que aparecen en el formulario de los estudiantes. Otros criterios son diferentes, por ejemplo, lo relativo a la investigación que realiza el profesor. Se entiende por pares del profesor aquellos otros profesores que, a juicio del director de departamento, conocen mejor su desempeño. Usualmente se pide esta información a unos 4 ó 6 pares del profesor evaluado. Los formularios se llenan en forma anónima y los resultados se dan siempre en forma consolidada. Esto asegura la transparencia del proceso y evita herir susceptibilidades.

c) La información contenida en la autoevaluación del profesor. Al profesor se le pide que llene otro formulario, semejante a los anteriores. Este formulario contiene el conjunto de criterios que aparecen en los formularios que llenan los estudiantes y los pares. La comparación entre la opinión que tiene el profesor sobre sí mismo y la opinión que tienen otros sobre él relacionados con el mismo criterio y los mismos indicadores resulta ser muy útil en casos de contraste.

d) El análisis que hacen los directores de programas a los cuales el profesor presta servicios docentes. En la Universidad los profesores pertenecen a los departamentos, no a los programas (carreras de pregrado o posgrados). Los directores de los programas manejan los currículos y los planes de estudio, pero los programas no cuentan con profesores propios. Estos están ubicados en los departamentos. Por ello la evaluación periódica se realiza por el respectivo director de departamento. Resulta útil para esta labor que él conozca la opinión que tienen, sobre el profesor, los directores de los programas a los cuales el profesor presta servicios. Para este efecto no se ha creído necesario ni oportuno elaborar algún formato específico.

e) El análisis que adicionalmente haga el mismo director de departamento y los decanos de la facultad sobre el desempeño del profesor. Además de la información recibida de terceros, el director de departamento y los decanos también tienen una opinión personal sobre el desempeño del profesor, tanto en lo que se refiere a su docencia como a su investigación y servicio y a las labores de gestión, y en general sobre su contribución a los logros de objetivos en la facultad y en la Universidad. El director y los decanos conjugan esta opinión personal con la que reciben de otras fuentes.

f) Los demás elementos que aporte el profesor y que crea relevantes para el proceso de su evaluación periódica. Sobre este punto se hablará más adelante.

g) El cumplimiento de los elementos contenidos en el Acta de Compromiso, resultado de la evaluación periódica anterior. Sobre el Acta de Compromiso también se hablará más adelante. 
h) El plan de trabajo del profesor. El desempeño del profesor se analiza a la luz del plan de trabajo que se elabora conjuntamente entre el director de departamento y el profesor al comienzo de cada semestre.

\section{Expedientes de la evaluación}

El proceso de la evaluación periódica se respalda en los siguientes expedientes:

a) El Portafolio del Profesor. Este portafolio debe ser llevado por el propio profesor. En él, el docente consigna las copias de los diplomas de títulos universitarios, los certificados de cursos no conducentes a títulos, las certificaciones sobre experiencias académica o profesionales que considere relevantes para su desempeño profesoral, copias de las obras de producción intelectual, su participación en actividades de investigación y de servicio, su desempeño en comités y otras actividades de gestión, certificaciones de otros logros, tales como la competencia alcanzada en un idioma no nativo o distinciones académicas y los demás elementos de su vida académica, profesional y personal que él crea relevantes. En cualquier momento, pero especialmente en el momento previo a su evaluación periódica, el profesor aporta el contenido de su portafolio al departamento para que sea consignado en su carpeta.

b) La Carpeta del Profesor. Esta carpeta se lleva en el departamento al cual está adscrito el profesor. En la carpeta se guarda toda la información sobre el profesor que pueda ser relevante para su evaluación periódica. Por ejemplo, en la carpeta se guardan los reportes sobre la información que han dado estudiantes y pares del profesor, así como el resultado de la autoevaluación, el análisis que han hecho sobre el profesor los directores de programas a los cuales les presta servicios, los resultados globales de las evaluaciones de obras de producción intelectual y de logros, todo lo que el profesor aporte para su evaluación proveniente de su portafolio, el acta de compromiso acordada al final de la anterior evaluación periódica de desempeño académico y el plan de trabajo del profesor.

c) El Acta de Compromiso. Se trata de uno de los más importantes componentes del sistema de evaluación. Esta acta, resultado final del proceso de la evaluación periódica, debe contener un plan de mejoramiento, con compromisos mutuos, es decir, con compromisos del profesor y también con compromisos institucionales para apoyar al profesor en el cumplimiento del plan de mejoramiento. 


\section{El acto de la evaluación periódica}

La evaluación periódica de desempeño académico debe ser realizada conjuntamente por los decanos de la facultad y el director de departamento correspondientes sin que esté presente el profesor. Con esto se persigue que ellos analicen con mucha tranquilidad toda la información existente en la carpeta del profesor y que discutan abierta y desprevenidamente toda aquella otra información que conozcan.

El resultado se consigna en una síntesis que se discute posteriormente, tan pronto como sea posible, en re el director del departamento y el profesor. En esta reunión, el profesor puede controvertir cualquier opinión si tiene evidencias de que no se ajusta a la realidad. Como resultado de esta discusión, debe llegarse a un acuerdo entre el profesor y el director de departamento sobre el plan de mejoramiento, que se consigna en el acta de compromiso.

Finalmente, el resultado de la evaluación se expresa en términos de "excelente", "bueno", "regular" o "malo" y se envía a la Vicerrectoría Académica para fines de digitación en el Registro Académico de Profesores (RAP) y la correspondiente asignación de puntos. Por cada semestre comprendido en el período evaluado se le otorgan al profesor 15 puntos si el resultado de la evaluación periódica de desempeño académico es "excelente" y 10 puntos si el resultado es "bueno".

Es bueno recalcar que el proceso está descentralizado. La Vicerrectoría Académica no interviene en la evaluación periódica de desempeño académico, ni en la definición del plan de mejoramiento, ni en el acta de compromiso, ni en su seguimiento. El papel de la Vicerrectoría Académica se limita a la definición general del sistema, a la definición de los responsables de implementarlo, a la definición del cronograma general que se debe seguir y a la definición y la elaboración de los instrumentos y, finalmente, a dar el visto bueno para la digitación de los puntos correspondientes. Pero las dueñas del proceso mismo de evaluación son las facultades.

\section{Evaluación de desempeño administrativo}

En la Universidad Javeriana, a diferencia de lo que ocurría hace 10 años, el nombramiento en un cargo directivo en unidades académicas (vicerrector, decano, director de departamento o de programa o de instituto) no es considerado ni una promoción ni un ascenso. La promoción del profesor consiste en su ascenso en su carrera académica, es decir, su ascenso en el escalafón profesoral. Los cargos directivos son ejercidos por los profesores por un período predeterminado 
En la medida en que las evaluaciones conducen al mejor aprovechamiento de las fortalezas institucionales y de las personas que componen la comunidad universitaria, así como a la corrección de debilidades, y por ende conducen a las acreditaciones de programas y a las posibilidades de ascenso en el escalafón profesoral y de promoción en el caso de personal administrativo, se logra una disposición muy favorable de las personas a su evaluación personal y a la evaluación de las dependencias y programas en que se desempeñan. en los reglamentos de la Universidad, al final del cual regresan a la condición de "profesor", en la categoría del escalafón que tienen y, por lo tanto, a ejercer plenamente la docencia, la investigación y el servicio. Por lo anterior, el interés principal del profesor es su carrera académica, no su nombramiento en algún cargo directivo.

El cargo directivo implica una remuneración adicional que desaparece cuando finaliza el período de ese cargo. Los profesores sienten que esta remuneración no compensa las menores oportunidades de realizar obras de producción intelectual y por lo tanto de obtener los puntos necesarios para el ascenso. Por este motivo, la remuneración adicional por un cargo directivo, por sí sola, no justifica el retraso en la carrera académica que le significa al profesor el ejercicio de dicho cargo. Por esto el Reglamento del Profesorado prevé que en las eva-

luaciones de desempeño administrativo se le otorguen puntos al profesor para que suplan, al menos en parte, los puntos que no se obtuvieron por la menor realización de obras de producción intelectual. Desde luego, el objetivo fundamental de la evaluación de desempeño administrativo no es el de otorgar puntos a los profesores para su ascenso en el escalafón. El objetivo fundamental es, como en las demás evaluaciones, contribuir al mejoramiento del desempeño administrativo.

Las evaluaciones de desempeño administrativo se realizan en forma paralela a las evaluaciones de desempeño académico del profesor y generan puntos, como ya se indicó, cuando el resultado es "excelente" o "bueno". Estas evaluaciones deben ser realizadas cada año y se otorgan 30 puntos por año por un resultado calificado como "excelente" y 20 puntos por un resultado calificado como "bueno".

Corresponde al jefe inmediato del directivo realizar las evaluaciones de su desempeño administrativo. El jefe que evalúa se basa en la información que se recoge de aquellas personas que están en capacidad de aportar algo. Para ello se han 
diseñado instrumentos muy semejantes a los que se utilizan en el caso de las evaluaciones de desempeño académico.

La evaluación de decanos académicos y de directores de departamento, carrera e instituto se iniciaron hace algo más de un año. Para la evaluación del vicerrector académico se están elaborando los respectivos formatos mediante los cuales se obtendrá la información que le permitirá al rector realizar dicha evaluación.

\section{Evaluación de obras de producción intelectual}

Las obras de producción intelectual se evalúan por pares académicos designados por el vicerrector académico para tal efecto. Las obras de producción intelectual, susceptibles de recibir puntos para fines de ascenso en el escalafón y de bonificación, se presentan en el Cuadro 1, con los correspondientes puntajes máximos que se pueden otorgar por cada obra:

\section{Cuadro 1}

Valoración de las obras de producción intelectual. Universidad Javeriana

\begin{tabular}{|l|c|}
\hline Obra & Máximio de \\
\hline Libros & 150 \\
Textos universitarios & 100 \\
Textos escolares & 70 \\
Manuales universitarios & 50 \\
Ensayos & 40 \\
Artículos en revistas indexadas intemacionalmente & 60 \\
Artículos en revistas nacionales científicas & 40 \\
Capítulos en libros colectivos & 40 \\
Ponencias en eventos científicos & 20 \\
Obras artísticas & 150 \\
Inventos & 150 \\
\hline
\end{tabular}

Adicionalmente, por la traducción de una obra ajena, se puede conceder hasta el 50\% de los puntos que le corresponderían a la obra según su tipo.

Para que a una obra se le pueda otorgar el máximo de los puntos correspondientes a su tipo, se requiere que sea calificada por los evaluadores como una obra "excelente" y que su difusión haya sido "internacional". El Cuadro 2 ilustra las categorías con las que se califican estas y el porcentaje máximo de puntos que puede ser otorgado por una obra según su calificación y ámbito de difusión. 


\section{Cuadro 2}

Criterios para la calificación de obras de producción intelectual. Universidad Javeriana. Los valores expresan el porcentaje de los puntos máximos que se pueden otorgar según el tipo de obra, indicados en el Cuadro 1.

\begin{tabular}{|l|c|c|c|}
\hline \multirow{2}{*}{ Calificación } & Ambito de reconocimiento académico de la obra \\
\cline { 2 - 4 } cualitativa & Internacional & Nacional \\
\hline Excelente & hasta $100 \%$ & hasta $80 \%$ & hasta $60 \%$ \\
Bueno & hasta $60 \%$ & hasta $50 \%$ & hasta $40 \%$ \\
Regular & hasta $20 \%$ & hasta $15 \%$ & hasta $10 \%$ \\
Malo & $0 \%$ & $0 \%$ & $0 \%$ \\
\hline
\end{tabular}

\section{Procedimientos}

El profesor debe inscribir la obra ante el decano académico de la facultad. Para ello llena un formato con los datos pertinentes y entrega una copia de la obra. El decano académico solicita al vicerrector académico el nombramiento de los pares que han de evaluar la obra y sugiere los candidatos. El vicerrector académico hace el nombramiento de los pares y les pide recoger la obra y la ficha de inscripción en la decanatura de la facultad.

Se nombran dos pares para la evaluación de cada obra. Ellos realizan su trabajo en forma individual. El par juzga, en primer lugar, si esta califica como obra de producción intelectual según los parámetros señalados. En segundo lugar, el par define la calificación de la obra en términos de "excelente", "buena", "regular" o "mala". En tercer lugar, define el ámbito de difusión de la obra. Finalmente, con base en todo lo anterior, otorga los puntos que la obra merece y envía el resultado a la vicerrectoría académica para su digitación

Hay tres resultados posibles de la evaluación:

a) Si la obra se califica positivamente con puntos por los dos evaluadores, los puntos otorgados por cada evaluador se promedian y el resultado corresponde al puntaje finalmente otorgado.

b) Si la obra se descalifica por los dos evaluadores, no se otorgan puntos. 
c) Si uno de los dos evaluadores califica la obra y el otro evaluador la descalifica, el decano académico debe solicitar al vicerrector académico el nombramiento de un tercer par. Si este califica la obra, los puntos otorgados se promedian con los del evaluador que originalmente había dado puntos por la obra. Si, en cambio, el tercer evaluador la descalifica, no sele otorgan puntos.

En cualquiera de los tres casos, el autor de la obra puede pedir una revisión de su evaluación. En este caso se procede al nombramiento de un nuevo evaluador, a quien se le da a conocer el resultado emitido por cada uno de los evaluadores previos. Este nuevo evaluador da un dictamen final y, en tal caso, lo que se digita es el resultado de su juicio.

Los puntos por producción intelectual no solo sirven para fines de ascenso. Adicionalmente se concede al autor una bonificación en dinero, por una sola vez por cada obra. El rector define el valor del punto para fines de bonificación cada año; por ejemplo, el valor del punto para las obras producidas durante 2003 fue de una suma de pesos equivalente a 3 dólares.

\section{Evaluación de logros}

La evaluación de logros consiste en la valoración que hace el decano académico de la facultad de la importancia del logro dentro de la carrera académica del profesor. Esta valoración conduce a la asignación de puntos, que le sirven al profesor para su eventual ascenso en el escalafón. Los logros por los cuales se pueden asignar puntos son:

- Segundos títulos universitarios

- Cursos formales no conducentes a título

- Distinciones académicas

- Competencia en idioma no nativo

- Traducción de obras del profesor a otros idiomas, realizada por terceros

Los procesos y criterios de puntuación para cada categoría de logro se describen a continuación.

\section{Segundos títulos universitarios}

En primer lugar, el Decano Académico define si el segundo título es de "tipo 1" ó de "tipo 2". Son títulos de "tipo 1" aquellos que son muy relevantes para las funciones que desempeña el profesor en la facultad y son de "tipo 2" los 
demás títulos obtenidos por el profesor. Los títulos obtenidos en Filosofía o en Educación se consideran siempre como "tipo 1" sin importar el departamento o la facultad en la que está ubicado el profesor.

En el Cuadro 3 se presentan los máximos puntajes que se otorgan por segundos títulos de "tipo 1" y "tipo 2".

Cuadro 3

Valoración de segundos títulos universitarios. Universidad Javeriana

\begin{tabular}{|c|c|c|}
\hline \multirow[b]{2}{*}{ Catcgonira del soggundo titulo } & \multicolumn{2}{|c|}{$\begin{array}{l}\text { Mádino de puntos } \\
\text { otorgados por trulo }\end{array}$} \\
\hline & 10100 & $\operatorname{lip} 02$ \\
\hline Pregrado & 60 & 30 \\
\hline Especialización (primera y siguientes) & 20 & 10 \\
\hline Maestría & 80 & 40 \\
\hline Doctorado & 160 & 80 \\
\hline Experiencia posdoctoral certificada & 50 & 25 \\
\hline
\end{tabular}

\section{Cursos formales no conducentes a título}

En primer lugar el Decano Académico define si se trata de un curso de "tipo 1" o de "tipo 2" bajo los mismos criterios anteriormente señalados. En segundo lugar, tiene en cuenta el número de horas del curso. Finalmente, define los puntos según los siguientes parámetros:

- Por un curso de "tipo 1", de 50 horas o más, puede otorgar hasta 10 puntos. Si el curso es de "tipo 2", hasta 5 puntos.

- Por un curso de "tipo 1", de menos de 50 horas, puede otorgar hasta 6 puntos.

Si el curso es de "tipo 2", hasta 3 puntos.

El total de puntos que se pueden otorgar a un profesor por cursos formales no conducentes a título, durante su permanencia en una de las categorías del escalafón, no puede ser superior a 50. Cuando el profesor ha acumulado 50 puntos dentro de su permanencia en una categoría por cursos no conducentes a título, solo se le pueden volver a otorgar puntos por este concepto cuando asciende de categoría. Pero, para ello los cursos deben tener fecha posterior a la 
del ascenso, dado que, según el Reglamento del Profesorado, no es posible transferir puntos de una categoría a otra.

\section{Distinciones o premios académicos}

Por una distinción o premio académico, se pueden otorgar hasta 50 puntos. En este caso, el decano académico propone el número de puntos, pero corresponde al vicerrector académico definir en cada caso cuántos puntos se le otorgan al profesor. Las distinciones pueden ser otorgadas por la misma Universidad o por otras entidades, sean académicas o no.

\section{Traducción de obras del profesor a otros idiomas}

Por cada obra del profesor, traducida por terceros a otros idiomas puede el decano académico otorgarle hasta 40 puntos al profesor. Si un profesor traduce sus propias obras o de terceros, el caso no es considerado como un "logro" sino como una "obra de producción intelectual”, lo cual se valora, como ya se indicó, con hasta el 50\% de los puntos correspondientes al tipo de obra, según se especifica en los cuadros 1 y 2.

\section{Competencia en idioma no nativo}

Por la competencia en cada idioma no nativo, se le pueden otorgar al profesor hasta 40 puntos si la competencia es "avanzada" y hasta 15 puntos si la competencia es "intermedia". Por un mismo idioma un profesor no puede recibir más de 40 puntos, de tal suerte que si se le otorgan 15 puntos por una competencia intermedia y después alcanza la competencia avanzada, se le pueden otorgar en ese momento solo los 25 puntos que le faltan para completar los 40 previstos. Para la comprobación del nivel de competencia se ha elaborado una tabla que señala las equivalencias de los puntajes de diferentes certificaciones reconocidas internacionalmente. La certificación más conocida es la del resultado del examen TOEFL. Un puntaje de entre 173 (500 en la antigua escala) y 209 se acepta como un nivel intermedio de competencia y un puntaje de 210 (550 en la antigua escala) o más, como un nivel avanzado de competencia.

\section{La evaluación y el ascenso en el escalafón}

El escalafón contempla cuatro categorías: Profesor Instructor, Profesor Asistente, Profesor Asociado y Profesor Titular. Los salarios de los profesores están atados directamente a la categoría del profesor. 
Para la ubicación inicial del profesor en el escalafón, al momento de vincularse a la Universidad, se tienen en cuenta la experiencia universitaria previa, el título universitario más alto alcanzado y su producción intelectual. Nadie puede ser ubicado en la categoría de profesor titular en el momento de vincularse a la institución. A esta categoría solo se puede llegar por ascenso.

Para ser ubicado en la categoría de profesor asociado se requiere que el candidato tenga una experiencia universitaria previa equivalente al menos ocho años a tiempo completo, que tenga título de doctor y al menos 350 puntos de producción intelectual. Si no tiene el título de doctor, este requisito puede ser reemplazado con 400 puntos adicionales de producción intelectual.

Se ubica al profesor en la categoría de profesor asistente cuando tiene una experiencia universitaria previa equivalente a al menos tres años a tiempo completo, posee título de maestría o dos títulos de pregrado y al menos 120 puntos de producción intelectual. Si no tiene el título de maestría, este requisito puede ser reemplazado con 300 puntos adicionales de producción intelectual.

Para ser ubicado en la categoría de profesor instructor solo se requiere que el candidato tenga el título de pregrado.

Los nombramientos de los profesores de planta requieren de un concurso. Los profesores contratados por horas no se rigen por el escalafón y no se categorizan. En estos casos su nivel salarial se establece mediante otro sistema que pondera equilibradamente el título máximo del profesor, su experiencia académica y su experiencia profesional.

Los requisitos para los ascensos de los profesores de planta en el escalafón son los siguientes:

a) Para ascender a la categoría de profesor asistente se requiere una permanencia de al menos tres años en la categoría de profesor instructor, haber acumulado al menos 150 puntos provenientes de producción intelectual, evaluaciones de desempeño y evaluaciones de logros, tener título de maestría o dos pregrados, o suplir el requisito del título de maestría con 300 puntos adicionales.

b) Para ascender a la categoría de profesor asociado se requiere una permanencia de al menos cinco años en la categoría de profesor asistente, haber acumulado durante su permanencia en dicha categoría al menos 300 puntos provenientes de producción intelectual, evaluaciones de desempeño y evaluaciones de logros, tener título de doctor, o suplir el requisito de este título con 400 puntos adicionales.

c) Para ascender a la categoría de profesor titular se requiere una permanencia de al menos siete años en la categoría de profesor asociado, haber acumulado durante su permanencia en dicha categoría al menos 600 puntos provenientes de producción intelectual, evaluaciones de desempeño y evaluaciones de 
logros y tener título de doctor. En este caso el requisito de título no puede sustituirse con puntos. No obstante, cuando no hay en el país suficientes doctores en la carrera o disciplina del profesor, el Consejo Directivo Universitario puede aprobar que el requisito del título de doctor se supla por medio de un proceso de méritos que implica la elaboración de un trabajo, científico o artístico, elaborado especialmente para este efecto.

\section{Efectos del sistema de evaluación}

La puesta en ejecución del sistema de evaluación de los profesores ha hecho posibles importantes logros en la Universidad, aunque también se han generado algunos problemas como los que se enuncian a continuación:

Los principales logros han sido:

a) El mejoramiento de la calidad del cuerpo profesoral. Como resultado de la aplicación del reglamento del profesorado en general, del escalafón de profesores y del sistema de evaluación, ha habido incentivos paraque el cuerpo profesoral tenga hoy un perfil muy diferente al que existía hace diez años. La proporción de profesores de planta frente a los profesores por horas cátedra ha crecido significativamente ${ }^{5}$. Hoy ya no puede decirse que hay una alta dependencia en el profesorado por horas cátedra. Esto ha permitido la conformación de una comunidad profesoral científica e idónea. La proporción de profesores con doctorado y con maestría ha crecido muchísimo ${ }^{6}$. La investigación se ha incrementado notablemente y la producción intelectual, antes casi inexistente, ya es importante ${ }^{7}$.

b) El mejoramiento de las condiciones de trabajo. Los salarios son ahora competitivos y el ambiente que se respira es muy bueno. En la medida en que las evaluaciones han mostrado las necesidades de cambios institucionales, se han ido implementando mejoras.

c) El afianzamiento de la cultura de la evaluación. Se ha perdido el temor a la evaluación y la animadversión a ser evaluado. Los profesores han entendido que las evaluaciones no son un elemento de control sino que son fuente de

5 Actualmente hay más de 1.400 profesores de planta, la gran mayoría de ellos de tiempo completo y algunos pocos de medio tiempo, para una población estudiantil cercana a 19.000 estudiantes.

6 En la Universidad actualmente ya el 10\% de los profesores de planta tienen títulos de doctorado y $73 \%$ en total tienen títulos de posgrado. Existe un programa de formación en la Universidad. Dentro de este programa se han formado en los últimos 10 años 158 profesores en el exterior en programas de posgrado, 89 de ellos en programas de doctorado y 53 en programas de maestría (los demás en especializaciones). 
mejoramiento y por lo general agradecen que el mejor conocimiento de sí mismos les haya permitido crecer.

d) El logro de niveles cada vez más altos de excelencia académica. Producto de los procesos evaluativos se ha obtenido la acreditación de muchísimos programas y la acreditación de la institución como un todo. Fue la primera universidad del país en obtener la acreditación institucional, la cual fue otorgada por el Ministro de Educación Nacional, mediante una recomendación del Consejo Nacional de Acreditación, por un período de ocho años.

Entre los principales problemas encontrados, está el agotamiento por las múltiples instancias de evaluación. Hay cansancio, muy notorio en algunas facultades por tantas evaluaciones que se deben cumplir.

También se ha detectado una falta de cumplimiento estricto de los períodos de evaluación ordenados por el Reglamento.

En conclusión, a pesar de reconocer la existencia de problemas, puede decirse que el balance final es positivo. Sin embargo, está previsto que en el curso de los próximos años se realice una nueva metaevaluación de todo el sistema de evaluación.

\section{Referencia}

Pontificia Universidad Javeriana. (2000). Reglamento del profesorado. Bogotá. 